\title{
Derecho global Global law
}

\author{
Ángel Aday Jiménez Alemán \\ Universidade de Vigo \\ adayiimenez@uvigo.es
}

Recibido / received: 16/08/2016

Aceptado / accepted: 08/09/2016

DOI: http://dx.doi.org/10.20318/eunomia.2016.3290

\section{Resumen}

La globalización jurídica y la generación, aún embrionaria, de un derecho global son manifestaciones del multifacético fenómeno de la globalización, entendida como aceleración y fusión de las relaciones sociales hasta llegar a constituir un todo orgánico. La cada vez más abundante literatura alrededor de este fenómeno no ha permitido aún que se supere la etapa precientífica, especialmente en las disciplinas jurídicas. El derecho no ha sido ajeno a la desterritorialización, aceleración e intensificación de las relaciones sociales, produciéndose una nueva realidad desordenada y múltiple. La globalización jurídica que se viene manifestando, entre otros hechos, a través de la difusión y adopción de semejantes instituciones jurídicas entre ordenamientos jurídicos, cuando no su integración, junto con la transferencia de una mayor capacidad de decisión a órganos de naturaleza jurisdiccional en constante diálogo. A su vez, han proliferado órdenes jurídicos que, desde instancias distintas a las estatales, regulan relaciones sociales que superan el ámbito propio del Estado-nación, planteándose la existencia de un ordenamiento jurídico global.

\section{Palabras clave}

Globalización, derecho global, órdenes jurídicos transnacionales, globalización jurídica, pluralismo jurídico.

\begin{abstract}
The legal globalization and the generation of a global law -still in early stages- are expressions of the globalization as a multifaceted phenomenon, and understood as the acceleration and merging of social relationships until the constitution of a whole organic body. In spite of the overwhelming literature related to globalization, we have not go beyond a prescientific stage, especially in the legal sciences. Law has been affected as well by the deterritorialisation, acceleration and intensification of social relationships, emerging a new disordered and multiple reality. The legal globalization has been revealing itself by, among other facts, the diffusion and adoption of similar legal institutions in different legal orders, or even their integration, along with the transferring of power to jurisdictional organs in a constant dialogue. At the same time, we have been witnesses of the proliferation of transnational legal orders. From entities that are not the nation-states, they regulate social relationships beyond the jurisdiction of the nation-state, coming up the question about the existence of a global legal order.
\end{abstract}

\section{Keywords}

Globalization, global law, transnational legal orders, legal globalization, legal pluralism. 
SUMARIO. 1. Introducción: La globalización como contexto. 2. La globalización del Derecho. 3. El Derecho Global: su naturaleza y sus características. 4. A modo de conclusión.

1. Introducción: La globalización como contexto

Antes de abordar la cuestión del derecho global resulta más que conveniente contextualizarla como una de las manifestaciones del multifacético fenómeno de la globalización. Y es que no cabe duda acerca de la centralidad de este concepto tanto en nuestra época histórica como en las ciencias sociales, protagonismo sólo compartido y desde hace bien poco con el de desigualdad (en concreto desde la publicación de El Capital en el Siglo XXI de Thomas Piketty).

Es un término cacareado en los medios de comunicación hasta la náusea, omnipresente más allá de tendencias y, del mismo modo, lleva presente entre los objetos de estudio de los investigadores de forma explícita más de medio siglo, aproximadamente. Tal es así que hay quien afirma como Fontana (2003) que no es posible evitar a lo hora de desarrollar algún tipo de investigación en ciencias sociales hacer una mínima mención a la globalización, con el riesgo de banalización y de reproducción del vacío que ello supone. Sin embargo, y aplicando los conceptos de Kuhn (1971), aún es difícil afirmar que se haya superado la etapa precientífica a su alrededor. Se empiezan a alcanzar consensos, pero las teorías resultan incompletas, quizás en parte por la propia naturaleza de este objeto científico (hecho que como veremos más adelante se agrava en las disciplinas jurídicas). Lo cual también puede ayudar a comprender el recalcitrante halo de novedad que acompaña al término, aunque lleve tanto entre nosotros ${ }^{1}$.

Desde que McLughan acuñara la idea de la "aldea global" en 1964 y a partir de su definición, se ha ido incidiendo en las características del fenómeno, siendo sus ejes la aceleración y la fusión de las relaciones sociales hasta llegar a constituir un "todo orgánico"2. Los cambios radicales para las relaciones sociales que empezaban a ser patentes en la década de los 60 del siglo XX y que McLughan supo observar y teorizar, no han hecho sino intensificarse de forma paulatina, de ahí que su trabajo nos siga siendo clave, y no sólo como hito fundacional. Con graduaciones, el espacio, el tiempo y el territorio en el que se desarrollan las relaciones sociales se ha unificado permitiéndonos hablar del "todo orgánico" participado por infinidad de elementos interrelacionados en una intensidad diversa.

Así el consenso se ha ido articulando alrededor de la de la globalización como un fenómeno multifacético, que entre sus causas recoge al acelerado cambio tecnológico, la multiplicación de la actividad económica transfronteriza y la miríada de instrumentos con el objetivo de liberalizar las políticas económicas a nivel estatal, regional y mundial ${ }^{3}$; y que abarca y va más allá de lo económico, de lo político y de lo cultural. En él resulta especialmente difícil enunciar hipótesis de relaciones de causa y efecto, si no poco más allá de la identificación de interrelaciones de determinación y refuerzo de las tendencias que se dirigen en ese sentido: el de la globalización como una vis atractiva irrefrenable, que irremediablemente va ocupando más espacios de relación social.

\footnotetext{
${ }^{1}$ El primer uso de este término se suele atribuir a Modelsky (1972).

2 Sobre esta concepción, Mclughan (1992) y Mclughan y Powers (1993).

${ }^{3}$ Entre otros muchos estudios sobre esta cuestión se puede acudir al elaborado por Garret (2000).
} 
La globalización ocupa los espacios ya existentes y los nuevos que se generan, desterritorializando las relaciones sociales. Multitud de actividades humanas tienen lugar hoy en espacios que han trascendido al espacio meramente físico, superando todo tipo de límites geográficos, ya sean naturales o jurídicos, siendo irrelevante dónde se hallar quienes participan en esa relación social y quienes resultan por ella influenciados. Por lo tanto, la escala del Estado-nación deviene insuficiente para la comprensión de buena parte de los fenómenos sociales, incluidos los jurídicos, produciéndose una "desestabilización de las viejas jerarquías escalares", en palabras de Saskia Sassen (2007).

Junto con la desterritorialización de las relaciones sociales, y al mismo tiempo como causa y efecto, tenemos la intensificación de la interdependencia de las relaciones sociales, relaciones que, en su mayoría y hasta no hace mucho, quedaban dentro del marco del Estado-nación como organización social. Se abre así un nuevo espacio real de cooperación y conflicto necesario para atender a las necesidades humanas. $\mathrm{Y}$, a su vez, las relaciones sociales no sólo se desterritorializan y aparecen en un nuevo espacio, se intensifica su interdependencia, sino que además se aceleran. Interrelaciones personales de todo tipo y multitud de constantes flujos de personas, información, capitales y mercancías, realizándose buena parte a tiempo real, lo que incide de nuevo en la unidad de espacio y tiempo mundial. $Y$ todo ello en un proceso de larga duración, gradual, que se intensifica gracias a los avances tecnológicos de todo tipo y que se retroalimenta a través de la multiplicación e intensificación de las relaciones sociales a nivel global. La consecuencia es la práctica unidad del espacio y del tiempo, lo que determina, en diferente grado, la experiencia de más seis mil millones de seres humanos.

Por supuesto que este fenómeno hunde sus raíces en un periodo histórico anterior, por lo que la clave para su comprensión e identificación no reside en el momento de su origen, sino en el de su intensificación. A mediados del siglo XIX ya existían voces conscientes de esta situación, como la de Marx (2004), anunciando el surgimiento de una red comercial universal de bienes tanto materiales como espirituales. Sin embargo, es la intensificación de este proceso hasta el grado que nos permite hablar del todo orgánico lo que permite afirmar la existencia de un único espacio, tiempo y territorio que abarca todo el planeta, lo que caracteriza a la globalización hoy como fenómeno social multifacético. $Y$ entre estas facetas de la globalización, el derecho, parafraseando a Marx, pasaría a ser uno de esos productos espirituales que pasa a formar parte del acervo común global, un derecho-mundo parte a su vez de una cultura-mundo ${ }^{4}$ : el germen de un auténtico orden jurídico global común, que sin prescindir de los tradicionales ordenamientos jurídicos, articula y organiza las relaciones sociales en esa unidad espacial y temporal como auténtica "técnica social específica de un orden coactivo", tal y como Kelsen (1995: 22) definió el derecho.

\section{La globalización del Derecho}

Como decimos, el derecho no ha quedado ajeno a la globalización. No sólo lo económico y lo político han salido del marco conceptual del Estado nacional ${ }^{5}$. Todo lo contrario, el derecho es una de sus más claras manifestaciones, una manifestación del occidente globalizado, de su cultura-mundo y de sus valores y normas, un mundo, según Goldstein (2001) que se mueve hacia el derecho. La globalización o mundialización jurídica se viene construyendo en ciertos sectores y a base de golpes en diferentes momentos históricos, dinámica que se ha vigorizado a

\footnotetext{
${ }^{4}$ Sobre este concepto, ver Lipovetsky y Juvin (2011).

${ }^{5}$ Entre los múltiples estudios sobre la globalización, vid. BECK, U. (1998).
} 
partir de la Segunda Guerra Mundial ${ }^{6}$. Los avances tecnológicos y la aceleración del comercio sobre la base jurídica construida previamente en los espacios coloniales por las potencias europeas, que implantaron sus instituciones jurídicas en sus respectivos territorios y poblaciones de influencia, construyendo lo que conocemos como familias jurídicas, principalmente common law y civil law, han dado lugar a un fenómeno desordenado y múltiple.

Y por supuesto, este fenómeno también hunde sus raíces en la historia, y mucho antes de la segunda mitad del siglo XX. Aún hoy es palpable el traslado en el espacio de ideas e instituciones jurídicas originarias del common law, con el trasplante de principios constitucionales, forma de gobierno, protección de derechos fundamentales y sistema jurídico, tanto con respecto a la producción normativa como a postulados normativos concretos. En Blackstone (1765-1769: 105) podemos encontrar una narración detallada del mecanismo de este proceso. El colonizador inglés portaba consigo su derecho, y estaba legitimado a aplicarlo y adaptarlo a las circunstancias del nuevo entorno por tratarse de un territorio bien no habitado, o bien adquirido por conquista o tratado. Este actor ha de ser sustituido hoy por la empresa estadounidense, que desde la prominente posición de los Estados Unidos en la economía global durante la segunda mitad del siglo $\mathrm{XX}$, actúa como agente difusor del derecho mercantil angloamericano. Cuestión que va más allá del poder económico y de un cierto colonialismo jurídico, sino que entronca con la naturaleza de los ordenamientos jurídicos de la familia del common law ${ }^{7}$.

Y otra tendencia que también se viene produciendo en el marco de la globalización jurídica es el surgimiento y preponderancia de una comunidad global de tribunales. En el último siglo hemos pasado de un solo órgano judicial internacional, la Corte Permanente de Arbitraje, a decenas de ellos, como viene analizando Romano (2011). Y a partir de una multitud de órdenes jurídicos transnacionales especializados en ámbitos de regulación específico ${ }^{8}$, una estructura de gobierno mundial está surgiendo, una fuerza política unida por normas jurídicas, procedimientos y redes multilaterales. $Y$ en esta estructura global de gobernanza, el poder de toma de decisiones se encuentra en lo que se viene conociendo tras la denominación de Slaughter (2003) como "una comunidad global" de órganos jurisdiccionales internacionales y nacionales. Sin olvidar que, dentro del estricto ámbito estatal, apenas quedan Estados que no desarrollen formas de constitutional review ${ }^{9}$.

Todo ello supone que se ha transferido una gran capacidad de poder desde las instituciones de representación a órganos de naturaleza jurisdiccional en los niveles estatal y supranacional ${ }^{10}$. $Y$ visto en conjunto, estamos ante un auténtico nuevo orden mundial ${ }^{11}$, algo más allá del supuesto diálogo entre tribunales, parafraseando a Vergottini (2010). Por lo que, podemos afirmar, la globalización jurídica no se está llevando a cabo por un legislador universal, sino por una multitud

\footnotetext{
${ }^{6}$ Ver Delmas-Marty (1999: 22).

${ }^{7}$ Shapiro (1993) desarrolla esta cuestión, planteando la íntima relación entre la regulación mercantil del common law y la evolución del capitalismo y de la organización empresarial.

${ }^{8}$ Halliday y Shaffer (2015) incluyen ámbitos tan diversos como el comercio internacional, los mercados financieros, tributos e impuestos, la seguridad alimentaria, los productos farmacéuticos o el cambio climático.

${ }_{9}$ Ginsburg (2008) analizó las posibles causas de la universalización de la jurisdicción constitucional, identificando el temor de la clase hasta ese momento dirigente ante la competencia electoral y la pérdida de poder como el motivo principal para la adopción de tribunales constitucionales como mecanismos contramayoritarios.

${ }^{10}$ Hirschl (2007) conceptualizó este fenómeno como juristocracia, producto en parte del interés de las autoridades políticas en evitar su posicionamiento en cuestiones de escaso rédito electoral.

${ }^{11}$ Para un análisis detallado, Slaughter (2004).
} 
de jueces y tribunales en comunicación incesante. La gobernanza de la globalización se viene confiando a instituciones burocráticas aparentemente puras, legitimadas por sus conocimientos técnicos, ejerciendo un dominio lógico-racional weberiano, confiando buena parte de la capacidad de decisión, o al menos, las decisiones más importantes y la resolución de conflictos, a órganos de carácter jurisdiccional que han ido mucho más lejos del ideal del juez como buche de la loi de Montesquieu. Por lo tanto, hay una huida de los procedimientos de toma de decisiones democráticos a favor de procedimientos tecnocráticos, y sobre todo, a una forma de tecnocracia: la juristocracia.

Pero además globalización jurídica también implica la integración entre ordenamientos jurídicos. Por ejemplo, encontramos de forma destacada la convergencia o integración de las dos familias preponderantes, el common law y el derecho civil continental, convergencia que podemos entender como el proceso por el que diversos sistemas jurídicos se asemejan progresivamente, adoptando normas que se pueden identificar como comunes, ya sean sustantivas, adjetivas o incluso instituciones íntegras. Existe consenso doctrinal de que este fenómeno es una realidad al menos en el ámbito del Derecho privado en el espacio normativo común europeo. Espacio paradigmático y auténtico laboratorio, dado que hasta ahora no se había avanzado tanto hacia la integración de ordenamientos a nivel supranacional, y que ha venido ofreciendo una oportunidad de análisis de la integración entre ordenamientos de familias distintas. $Y$ a pesar del próximo abandono del Reino Unido de la Unión Europea, continuará ofreciendo una realidad digna de ser estudiada.

\section{El Derecho Global: su naturaleza y sus características}

Aparte de la cuestión de la globalización del derecho, no podemos dejar de abordar, aunque sea someramente, la generación muy incipiente de un derecho global. Como hemos afirmado anteriormente, tiempo y espacio se funden y se producen normas que rigen esa nueva unidad social: el derecho y su producción se desterritorializan, se desestatalizan y se privatizan, al menos en parte. Lo cual resulta paradójico si tenemos en cuenta la estrechísima relación entre territorio y derecho propia del derecho pregobal, la íntima relación entre la Tierra y el derecho descrita por Carl Schmitt (2005: 22) en su Nomos de la tierra. Una unión triple, en la que el derecho es premio al trabajo, límite y signo público del orden con fundamento en la Tierra.

Esta idea o bien se fractura o al menos se transforma como efecto de la globalización. El marco de globalización jurídica en el que nos situamos trasciende cualquier tipo de relación con el territorio y se caracteriza por normas y procedimientos jurídicos que rigen procesos políticos y económicos transnacionales que determinan a los procesos de producción normativa estatales. La intensificación contemporánea de este fenómeno nos permite hallar normas uniformes transnacionales, múltiples órdenes jurídicos, especialmente en el ámbito económico, como la nueva lex mercatoria ${ }^{12}$, los principios y convenciones del Institute for the Unification of Private Law (UNIDROIT) mundialmente seguidos y Lando en Europa, entre muchos otros.

Lo cual nos dirige hacia otra tendencia a tener muy en cuenta: En el contexto de la globalización, el paradigma kelseniano de unidad entre Estado y Derecho se diluye, al igual que el principio del Estado como punto último de imputación, como voluntad superior sobre la cual no hay ninguna otra. La validez del orden jurídico sigue descansando en el poder del Estado, pero tanto el orden jurídico como el

\footnotetext{
${ }^{12}$ Ver, entre otros, Galgano (2003)
} 
poder superan progresivamente sus límites estrictos. Por ello, ya no nos sorprende que buena parte de la creación del Derecho provenga hoy de ámbitos no estatales, enfrentándose de forma directa a la articulación estatal de la creación del derecho, distribuida entre funciones legislativa, judicial y ejecutiva, y aún más importante, a su fundamentación democrática. El "Leviathan 2.0", siguiendo el análisis de Maier (2012), el moderno Estado-nación que entre 1850-1940 operó como la organización política más eficiente, que no sólo monopolizó el ejercicio legítimo de la violencia sino también la identificación con el ordenamiento jurídico y de la producción normativa, se encuentra en un nuevo proceso de transformación.

Cuestión bien distinta es la de la generación de un auténtico ordenamiento jurídico global o transnacional, conceptos que venimos encontrando en la doctrina a la hora de aproximarse a fenómenos jurídicos que no encajan estrictamente como derecho internacional, pero sí que van más allá de los límites de las jurisdicciones estatales, y que implican la creación de normas en instancias no estatales y que se dirigen a la regulación de relaciones sociales que superan el ámbito que tradicionalmente se viene entendiendo como propio del Estado-nación.

Como efecto de la globalización, el paradigma y la tendencia a identificar el derecho que regula las relaciones sociales más allá de las jurisdicciones estatales desde que Bentham acuñase el concepto Derecho internacional ya no resulta operativa. En realidad, esta concepción no era mucho más que un derecho interestatal, como ha ido demostrado el acontecer de los tiempos y las transformaciones en las dinámicas de las relaciones internacionales. En los años cincuenta del siglo XX se vislumbraba el agotamiento del Derecho internacional como marco de regulación de todas las relaciones que se estaban produciendo más allá de las jurisdicciones estatales. Jessup (1956: 2) fue de los primeros que se encontró en la necesidad de utilizar el término "derecho transnacional", en lugar del de "derecho internacional", para referirse a todas las acciones o hechos regulados por el derecho que trascendieren fronteras estatales. Estas acciones y hechos podían implicar a individuos, empresas, Estados, organizaciones de Estados u otros colectivos. Y en la misma década el derecho internacional comenzaba a ser consciente de su imparable sectorización a pesar de su aspiración a ser el derecho común de la humanidad (Jenks, 1958). De la crisis del derecho internacional está surgiendo el derecho global, como afirma Oslé (2008), y para su análisis urge superar la dicotomía derecho estatal - derecho internacional, y lo idóneo es, siguiendo a Twinning (2003), el renacimiento de una teoría jurídica general.

Ni siquiera hay acuerdo en la conveniencia del término global a la hora de referirse a esta realidad jurídica. Halliday y Shaffer (2015: 4) apuestan por el uso de la denominación "transnacional", y no sin razón, dado que el derecho "global" supondría que la ordenación jurídica a la que hace referencia tendría que ser realmente global en su ámbito geográfico, cosa que aún no es. Dado que es cierto el ámbito de ese ordenamiento es diverso, en relación con el objeto de la regulación, lo geográfico y las organizaciones a las que afecta, incluyendo sujetos de derecho público y privado, el concepto "ordenes jurídicos transnacionales" sería más apropiada.

Los debates doctrinales no se quedan en cuestiones conceptuales, sino que van al grado de desarrollo del derecho global o transnacional. Algunos autores como Cassese (2006) no han dudado en afirmar que ya nos encontramos ante un auténtico ordenamiento jurídico global, aunque a los ojos de Romano (1963) bien nos costaría identificarlo. Allot (2007) también se encuentra en una posición próxima, afirmando sin ambages que estamos ante el surgimiento de un sistema jurídico universal, producto de la internacionalización de lo nacional, la 
nacionalización de lo internacional, y la universalización creciente de la regulación de cuestiones hasta no hace mucho propias de los sistemas jurídicos estatales. Aún no podemos afirmar que todo el orbe se halle bajo un único conjunto normativo y menos aún que esta haya sido producto de un único ente con capacidad de imponerlo o fruto del consenso de los entes políticos actuales o del mero devenir. La situación a día de hoy es infinitamente más compleja. La realidad es la de un conjunto de órdenes normativos muy diverso que nos permite identificar una serie de tendencias y características.

En la ausencia de una auténtica organización política supraestatal como autoridad superior, lo más que se podría afirmar es el desarrollo de una muy originaria fase de constitución de un ordenamiento jurídico supraestatal compuesto por múltiples órdenes jurídicos. Este ordenamiento jurídico global se basa más en la cooperación que en la soberanía, más en las redes que en las jerarquías, en la pluralidad de actores descentralizados que regulan sectores, en lugar de una única autoridad central. Un derecho fracturado, que fluye, permeable y policéntrico, de acuerdo a las características de Backer (2012), una suerte de sistematización de la anarquía, un regreso del pluralismo jurídico, que nunca se había terminado de ir, un espacio en el que conviven jurisdicciones territoriales y personales, en el que un cierto grado de conflictividad entre jurisdicciones es inevitable, pudiendo incluso llegar ser positivo tal y como lo observa Berman (2009).

\section{A modo de conclusión}

Frente a quienes aún niegan la realidad jurídica global o transnacional, blandiendo como argumento principal que en el espacio trasnacional sólo podemos encontrar una proyección de las relaciones de actores con sede en jurisdicciones estatales, la realidad es que estas relaciones se regulan por un rico, complejo y múltiple cuerpo normativo, que no puede ser entendido con las tradicionales conceptualizaciones de lo "estatal" y de lo "internacional", como ha observado entre otros Hongju Koh (2001).

Marcel Merle (1982) advirtió sobre la incómoda posición en la que se encuentra el jurista ante la realidad internacional, quien está dirigido irremediablemente a, por un lado, describir la práctica seguida por los sujetos de derecho, y por otro, a construir sistematizaciones que logren comprehender la totalidad de las relaciones sociales analizadas. Esta situación no ha hecho sino agravarse ante la sociedad internacional contemporánea, en la que se han desarrollado intensamente las tendencias de la década de los setenta del siglo pasado, una sociedad internacional en la que los Estados observan cómo paulatinamente las relaciones internacionales dejan de ser puramente relaciones interestatales, y comparten escena e incluso protagonismo con una pléyade de actores de todo tipo, aumentando la perplejidad del jurista contemporáneo de formación estatocéntrica y positivista. Sobre todo para el positivista del siglo XX que se ha formado, ejerce y forma nuevos juristas aceptando al Estado en el centro de su "cosmovisión jurídica".

Sin embargo, el derecho global, no se fundamenta en una organización política, y es entendido como un conjunto no definido de órdenes jurídicos, algunos de tendencia universal, pero en su mayoría dirigidos hacia sectores concretos, que, desde instancias distintas a las Estatales, regulan relaciones sociales que superan el ámbito propio del Estado-nación. Lo que lleva a algunas paradojas del jurista ante la juridificación intensa de las relaciones internacionales que está suponiendo la globalización, incluso la transformación del principio de Ulpiano ubi societas, ibi ius, en ubi ius, ¿ibi societas? Y lo sitúa ante la necesidad de una auténtica constitucionalización de la nueva realidad jurídica. 
Bibliografía

ALLOT, P. (2007), "The emerging universal legal system". En: NIJMAN J. E. y NOLLKAEMPER A., New Perspectives on the Divide Between National and International Law, Oxford, Oxford University Press, pp. 63-83.

BACKER, L. C. (2012), "The Structural Characteristics of Global Law for the 21st Century: Fracture, Fluidity, Permeability, and Polycentricity", Tilburg Law Review, núm 17, pp. 177-199.

BECK, U. (1998), ¿Qué es la globalización? Falacias del globalismo, respuestas a la globalización, Paidós, Barcelona.

BERMAN, P. S. (2009) "The New Global Legal Pluralism", Annual Review of Law and Social Science 2009, núm. 5, pp. 225-242.

BLACKSTONE, W. (1765-1769), Commentaries on the Laws of England, Clarendon Press, Oxford, disponible en http://avalon.law.yale.edu/subject menus/blackstone.asp.

CASSESE, S. (2006), La globalización jurídica, Marcial Pons-INAP, Madrid.

DELMAS-MARTY, M. (1999), La mondialisation du droit: chances et risques, Recueil Daloz, Paris.

MIN SL , R. (2008), ?, Thomson Aranzadi, Cizur Menor.

FONTANA, J. (2003), La globalización en una perspectiva histórica, Universidad de Oviedo D. L., Oviedo.

GALGANO, F. (2003), "Lex Mercatoria, Shopping del derecho y regulaciones contractuales en la época de los mercados globales", Revista de erecho Mercantil, núm. 247, pp. 7-20.

GARRET, G. (2000), "The Causes of Iobalization", Comparative Political Studies, Vol. 33, núm. 6/7, pp. 941-991.

GINSBURG, T. (2008), "The lobal Spread of Constitutional Review". En: WITTINGTON K. y KELEMAN, D. (eds.) The Oxford Handbook of Law and Politics, Oxford, Oxford University Press.

GOLDSTEIN, J. L. (2001), Legalization and World Politics (International Organization Special Issues), MIT Press, Cambridge.

HALLIDAY, T.C. y SHAFFER, G. (2015), Transnational Legal Orders, Cambridge University Press, Cambridge.

HIRSCHL, R. (2007), Towards Juristocracy: The Origins and Consequences of The New Constitutionalism, Harvard University Press, Cambridge.

H N JU K H, H. (2001), "The Iobalization of Freedom", The Yale Journal of Internacional Law, vol. 26, pp. 305-312.

JENKS, C.W. (1958), The Common Law of Mankind, Frederick A. Praeger, New York.

JESSUP, P.C. (1956), Transnational Law, Yale University Press, New Heaven.

KELSEN, H. (1995), Teoría General del Derecho y del Estado, Universidad Nacional Autónoma de México, México D. F.

KUHN, T. S. (1971), La estructura de las revoluciones científicas, Fondo de Cultura Económica, México D. F.

MAIER, CH. S. (2012), Leviathan 2.0: Inventing Modern Statehood, The Belknap Press of Harvard University Press, Cambridge.

MARX, K. y ENGELS, F. (2004), El manifiesto comunista, Akal, Madrid.

MCLUGHAN, M. (1992), Comprender los medios de comunicación: Las extensiones del ser humano, Paidós, Barcelona.

MCLUGHAN, M. y POWERS, B. R. (1993), La aldea global, Gedisa, Barcelona.

MERLE, M. (1982), Sociología de las relaciones internacionales, Alianza Editorial, Madrid.

MODELSKY, G. (1972), Principles of World Politics, Free Press, Nueva York. 
LIPOVETSKY, G. y JUVIN, H. (2011), El Occidente globalizado: Un debate sobre la cultura planetaria, Anagrama, Madrid.

R MAN , C. P. R. (2011), "The Proliferation of International Judicial Bodies: The Pieces of the Puzzle", International Law and Politics, Vol. 31, pp. 709-751.

ROMANO, S. (1963), El ordenamiento jurídico, Instituto de Estudios Políticos, Madrid.

SASSEN, S. (2007), Una sociología de la globalización, Katz Editores, Buenos Aires.

SCHMITT, C. (2005), El nomos de ti : En $d$ nt $d$ "I $p$ i $m$ p $m$ ", Editorial Struhart y Cía., Buenos Aires.

SHAPIRO, M. (1993), "The Globalization of Law", Indiana Journal of Global Legal Studies: Vol. 1, núm. 1, pp. 37-64.

SLAUGHTER, A.-M. (2003), "A lobal Community of Courts", Harvard International Law Journal, vol. 44, núm 1, pp. 192-218.

SLAUGHTER, A.-M. (2004), A New World Order, Princeton University Press, Princeton.

VERGOTTINI, G. (2010), Más allá del diálago entre tribunales: Comparación y relación entre jurisdicciones, Editorial Aranzadi, Cizur Menor.

WEBER, M., (2002), Economía y sociedad, Fondo de Cultura Económica, México D. $\mathrm{F}$.

TAMANAHA, B. Z., (2007), Understanding Legal Pluralism: Past to Present, Local to Global, Sydney Law Review, vol. 30, pp. 375-411.

TWINNING, W. (2003), Derecho y globalización, Siglo del Hombre Editores, Instituto Pensar, Universidad de los Andes-Facultad de Derecho, Bogotá. 